\title{
High-speed integrated nanowire circuits
}

Inexpensive sophisticated circuitry can be 'painted' on to plastic or glass substrates.

M

acroelectronic circuits made on substrates of glass or plastic could one day make computing devices ubiquitous owing to their light weight, flexibility and low cost ${ }^{1}$. But these substrates deform at high temperatures so, until now, only semiconductors such as organics and amorphous silicon ${ }^{2}$ could be used, leading to poor performance. Here we present the use of low-temperature processes to integrate high-performance multi-nanowire transistors into logical inverters and fast ring oscillators on glass substrates. As well as potentially enabling powerful electronics to permeate all aspects of modern life, this advance could find application in devices such as low-cost radio-frequency tags and fully integrated high-refresh-rate displays.

The mobility of single-crystal semiconducting nanowires is comparable to that of computer-grade silicon ${ }^{3}$. Multi-nanowire transistors ${ }^{4,5}$ - analogous to thin-film transistors - can be assembled from solution on pieces of glass and plastic ${ }^{6}$. For many applications, however, fully interconnected nanowire devices that function as viable circuit elements operating at high frequencies will be required.

We integrated two nanowire thin-film transistors to generate inverters (Fig. 1a), which we made in a parallel process over glass substrates by using standard photolithography techniques (for methods, see supplementary information). The process gives a high yield of devices that show reliable, well defined signal inversion under direct current conditions (see supplementary information). Investigation of the alternating-current response of these inverters (Fig. 1b) shows that the gain, or signal amplification, is greater than unity, and the expected phase inversion is achieved when these devices are driven by a $1-\mathrm{MHz}$ sine wave at a supply of $15 \mathrm{~V}$. As signal propagation in an integrated system requires gain that is greater than unity, these results and the high reproducibility of our nanowire transistors suggest that fully interconnected nanowire oscillators could operate in the megahertz regime.

Our ring oscillators consist of three inverters in series (Fig. 1c), where the input of each inverter is connected to the output of the previous device, with a feedback loop to complete the ring. The necessary on-chip integration is achieved during fabrication and does not require any external wiring. We characterized the nanowire ring oscillators on glass substrates and found that the output-voltage oscillations were stable and self-sustained.

The devices show a maximal oscillation frequency of $11.7 \mathrm{MHz}$, corresponding to a

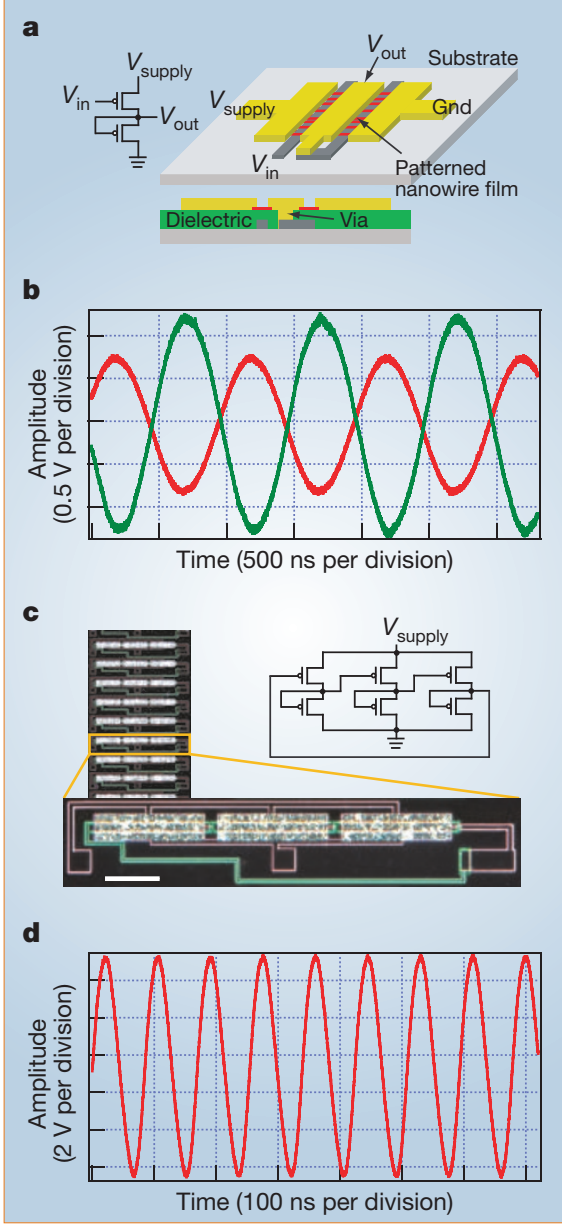

Figure 1 Alternating-current properties of integrated multi-nanowire circuits on glass. a, Circuit diagram and schematics of the multinanowire inverters. Labelled voltages are bias $\left(V_{\text {supply }}\right)$, input $\left(V_{\text {in }}\right)$ and output $\left(V_{\text {out }}\right)$ voltage. The dielectric is omitted in the perspective schematic for clarity. 'Via' indicates one of a pattern of holes in the dielectric layer that are used to connect different metal layers. b, Output waveform (green) of an inverter fabricated on glass driven by a $1-\mathrm{MHz}$ sine wave (red) with $V_{\text {supply }}=15 \mathrm{~V}$. c, Optical images and circuit diagram of nanowire ring oscillators. The gate level edge, source-drain level edge and nanowires appear green, pink and white, respectively, in dark field. Scale bar, $100 \mu \mathrm{m}$. d, Oscillation of $11.7 \mathrm{MHz}$ in a ring oscillator structure with $V_{\text {supply }}=43 \mathrm{~V}$.

stage delay of $14 \mathrm{~ns}$ (Fig. 1d). Significantly, all devices measured on glass have oscillation frequencies at or above $10 \mathrm{MHz}$. Furthermore, nanowire oscillators made on glass substrates have higher frequencies than devices made on silicon substrates (see supplementary information). This could be a key advantage for nanowire circuits as the properties of devices made with other materials often degrade upon transfer to non-crystalline substrates ${ }^{7}$.

The stable oscillation frequencies seen for our nanowire-based devices are many orders of magnitude larger than previous ring oscil- lators based on nanoscale building blocks. For example, carbon-nanotube devices have oscillation frequencies from 5 to $220 \mathrm{~Hz}$ (refs 8,9). Although this is not an intrinsic limit for nanotubes, it highlights how important reproducible material properties are for the successful creation of integrated, highperformance devices.

It is interesting to compare these nanowire-device features with those of organic ring oscillators, given that the active material in both can be deposited at ambient temperatures from liquid solutions. Reported stagedelay times for organic ring oscillators are typically longer than 300 ns (ref. 10) and therefore substantially $(20 \times)$ slower than those we obtain for nanowires on glass. Comparable results have been seen for other semiconductors with low synthesis temperatures; to our knowledge, the fastest reported stage delay for amorphous silicon ring oscillators is $210 \mathrm{~ns}$ (ref. 11).

Our integrated nanowire-based transistors open the way to a variety of electronic applications; the techniques we describe are all compatible with low-deformation-temperature materials such as plastics, broadening the scope for design. One limitation is that supply voltages of 35 volts or more are required to achieve stable oscillations, but device structures could be improved by incorporating higher- $k$ dielectrics, more advanced nanowire materials and reduced channel lengths. This would enable them to be operated at lower voltages and much higher frequencies, taking low-cost electronics to high-performance computing levels.

Robin S. Friedman ${ }^{\star}$, Michael C. McAlpine*, David S. Ricketts $\dagger$, Donhee Ham $\dagger$,

\section{Charles M. Lieber ${ }^{\star} \dagger$}

${ }^{\star}$ Department of Chemistry and Chemical Biology,

$\dagger$ Division of Engineering and Applied Sciences,

Harvard University, Cambridge,

Massachusetts 02138, USA

e-mail:cml@cmliris.harvard.edu

1. Gelinck, G. H. et al. Nature Mater. 3, 106-110 (2004).

2. Street, R. A. Technology and Applications of Amorphous Silicon (Springer, New York, 2000).

3. Cui, Y., Zhong, Z., Wang, D., Wang, W. U. \& Lieber, C. M. Nano Lett. 3, 149-152 (2003).

4. Jin, S. et al. Nano Lett. 4, 915-919 (2004)

5. Duan, X. et al. Nature 425, 274-278 (2003).

6. McAlpine, M. C. et al. Nano Lett. 3, 1531-1535 (2003).

7. Shimoda, T., Inoue, S. \& Utsunomiya, S. Proc. SPIE 4295, 52-59 (2001).

8. Bachtold, A., Hadley, P., Nakanishi, T. \& Dekker, C. Science 294, $1317-1320$ (2001).

9. Javey, A., Wang, Q., Ural, A., Li, Y. \& Dai, H. Nano Lett. 2, 929-932 (2002).

10. Clemens, W., Fix, W., Ficker, J., Knobloch, A. \& Ullmann, A. J. Mater. Res. 19, 1963-1973 (2004).

11. Hiranaka, K., Yamaguchi, T. \& Yanagisawa, S. IEEE Electron Device Lett. 7, 224-225 (1984).

Supplementary information accompanies this communication on Nature's website.

Competing financial interests: declared none. 